\title{
BAROBAMPO C2015, NUEVA VARIEDAD DE TRIGO CRISTALINO PARA ÁREAS DE RIEGO EN MÉXICO
}

\author{
BAROBAMPO C2015, A NEW DURUM WHEAT CULTIVAR \\ FOR IRRIGATED AREAS IN MEXICO
}

\begin{abstract}
Héctor E. Villaseñor-Mir ${ }^{1}$, Julio Huerta-Espino ${ }^{1}$, René Hortelano-Santa Rosa ${ }^{1}$, Eliel MartínezCruz $^{1 *}$, María F. Rodríguez García', Ernesto Solís-Moya ${ }^{2}$, Jorge I. Alvarado-Padilla ${ }^{3}$, Alberto Borbón Gracia ${ }^{4}$, Gabriela Chávez-Villalba ${ }^{4}$, Héctor Cortinas-Escobar ${ }^{5}$, Leodegario OsarioAlcalá $^{6}$, Eduardo Espitia-Rangel ${ }^{1}$, Juan Martínez-Medina ${ }^{7}$ y Javier Ireta-Moreno ${ }^{8}$
\end{abstract}

\begin{abstract}
'Instituto Nacional de Investigaciones Forestales, Agrícolas y Pecuarias (INIFAP), Campo Experimental Valle de México, Coatlinchán, Texcoco, Estado de México, México. ${ }^{2}$ INIFAP, Campo Experimental Bajío, Celaya, Guanajuato, México. ${ }^{3}$ INIFAP, Campo Experimental Valle de Mexicali, Mexicali, Baja California, México. ${ }^{4}$ INIFAP, Campo Experimental Norman E. Borlaug, Ciudad Obregón, Sonora, México. ${ }^{5}$ INIFAP, Campo Experimental Río Bravo, Rio Bravo, Tamaulipas, México. ${ }^{6} I N I F A P$, Campo Experimental Valles Centrales de Oaxaca, Santo Domingo Barrio Bajo, Etla, Oaxaca, México. ${ }^{7} I N I F A P$, Campo Experimental General Terán, Ex hacienda Las Anacuas, General Terán, Nuevo León, México. ${ }^{8}$ INIFAP, Campo Experimental Centro Altos de Jalisco, Tepatitlán, Jalisco, México.
\end{abstract}

*Autor de correspondencia (martinez.eliel@inifap.gob.mx)

La totalidad de la producción de trigo cristalino (Triticum durum Desf.) en México se obtiene en condiciones de riego (SIAP, 2018), donde los principales problemas del cultivo son la incidencia de royas y la escasez de agua durante el ciclo de producción. En particular, en el año 2014 se presentaron dos nuevas razas de roya amarilla (Puccinia striiformis f. sp. tritici Eriks.) que vencieron la resistencia de la mayoría de las variedades que se siembran en México (Solís et al., 2016); además, la roya de la hoja (Puccinia triticina) presentó una variante en el año 2017 que volvió susceptibles a las variedades sembradas en el noroeste del país (Pérez-López et al., 2017). En los dos casos se ha requerido de la aplicación de fungicidas para su control.

En el año 2016 la producción anual de trigo cristalino en México fue de 2 millones de toneladas, de las que casi 700 mil se emplearon para consumo nacional y el resto se exportó (CANIMOLT, 2016). Una opción para hacer frente a esta situación es generar nuevas variedades que conjunten alto rendimiento, tolerancia a las royas y buena calidad de grano. En este contexto, el programa de trigo del Instituto Nacional de Investigaciones Forestales, Agrícolas y Pecuarias (INIFAP) pone a disposición de los productores de trigo de riego en México la variedad Barobampo C2015, que es resistente a las nuevas razas prevalecientes de roya en México, de alto rendimiento y con buena calidad industrial.

La línea se generó en el programa de trigos macarroneros del Centro Internacional de Mejoramiento de Maíz y Trigo (CIMMYT) a partir de una cruza trilineal y de la aplicación posterior de los métodos de selección de familias masivas y pedigrí. La $F_{1}$ se sembró en el ciclo agrícola otoño-invierno (O-I) 2004-2005 en Ciudad Obregón, Sonora (OTOPY), la $F_{2}$ en 0-। 2005-2006 en Ciudad Obregón, Sonora (10Y), la $F_{3}$ en primavera-verano (P-V) 2006 (OM) en Atizapán, Estado de México, la $F_{4}$ en O-I 2006-2007 en Ciudad Obregón, Sonora (4Y), la $F_{5}$ en P-V 2007 en Atizapán, Estado de México (OM), la $F_{6}$ en O-I 2007-2008 en Ciudad Obregón, Sonora (4Y) y la $F_{7}$ en P-V 2008 en El Batán, Texcoco, Estado de México, donde se cosechó como línea (OB). La evaluación de la tolerancia a la roya amarilla de esta línea se llevó a cabo en Nanacamilpa, Tlaxcala, en el ciclo P-V 2011 y la selección 22 (22NAN) se sembró en 0-I 20112012 en Roque, Guanajuato, la que se cosechó en masa (OR) y se identificó con la siguiente genealogía:

\section{GODRIN/GUTROS//DUKEM/3/THKNEE11/4/DUKEM1// PATKA7/YAZI1/3/PATKA7/YAZI1/5/AJAIA12/ \\ F3LOCAL(SEL.ETHIO.135.85)//PLATA13/3/ADAMAR}

y el siguiente pedigrí:

CDSS04B00367T-0TOPY-10Y-0M-4Y-OM-4Y-0B-22NAN$\mathrm{OR}$

Barobampo C2015 es de hábito de crecimiento de primavera, de ciclo intermedio (121 d promedio a madurez fisiológica), de porte medio (promedio de $89 \mathrm{~cm}$ de altura de planta), tolerante al acame (Figura 1), presenta fuerte coloración de antocianinas en el coleóptilo, es de crecimiento erecto, con ausencia o muy baja frecuencia de plantas con hoja bandera curvada, la pigmentación antociánica de las aurículas de la hoja bandera es débil, con ausente o muy débil vellosidad en el nudo superior y la espiga es color blanco y de longitud mediana. La gluma, en el tercio medio de la espiga es de forma ovoide y con forma del hombro redondeada; su pico es de longitud corta, sin

Recibido: 23 de abril de 2019

Aceptado: 21 de enero de 2020 
curvatura y con vellosidad en la superficie externa. El grano es color ámbar, de forma ligeramente alargada, los pelos del pincel son de longitud media y con coloración ligera al aplicarle fenol.

La línea se evaluó a partir del ciclo 0-I 2010-2011 hasta el ciclo 0-I 2014-2015 por el INIFAP en sus ensayos nacionales de rendimiento de trigos cristalinos en 10 estados del país y en 92 condiciones de cultivo, donde superó en rendimiento promedio a las 10 variedades testigo con valores superiores entre 5.9 (Cemexi C2008) y 14.3 \% (Movas C2011). En riego normal su rendimiento promedio fue de $5.8 \mathrm{t} \mathrm{ha}^{-1}$ y superior en $6.1 \%$ a Cemexi C2008 y en $15.2 \%$ a Bataquez C2004 y Rio Colorado C2009. En condiciones de riego limitado (estrés hídrico en la etapa de llenado de grano) su rendimiento promedio fue 4.5 t ha $^{-1}$, superior en $3.9 \%$ a Anatoly C2011 y en $13.9 \%$ a Movas C2011; además, se comportó como una variedad estable y consistente. En las evaluaciones en los ciclos O-I y primavera-verano (P-V) de los años 2011 a 2015, esta nueva variedad presentó lecturas máximas de roya de la hoja de 5R, de roya amarilla en la hoja de 15MR y en la espiga de $10 \%$, por lo que actualmente es la variedad de trigo macarronero que posee más tolerancia a las razas de royas que prevalecen en México.

En las pruebas de calidad industrial presentó un peso hectolítrico promedio de $85 \mathrm{~kg} \mathrm{hL}^{-1}$, que es igual al de la mejor variedad testigo (Cirno C2008) y un porcentaje de granos vítreos mayor a 90 (Figura 2), cualidades que favorecen el rendimiento de sémola. El color amarillo del endospermo del grano presentó valores de b mayores a 24 unidades en colorímetro (Konica Minolta, Tokio, Japón), lo que permitirá obtener pastas alimenticias con la coloración apropiada para la industria y grano con calidad de exportación.

Por lo anterior, Barobampo C2015 se recomienda para siembra bajo condiciones de riego normal y riego limitado durante el ciclo de otoño-invierno en los estados de Sinaloa, Baja California Norte, Chihuahua, Coahuila, Nuevo León, Tamaulipas, Guanajuato y Michoacán. En los estados del noroeste, norte y noreste se recomienda en fechas de siembra tempranas a tardías, mientras que en los estados
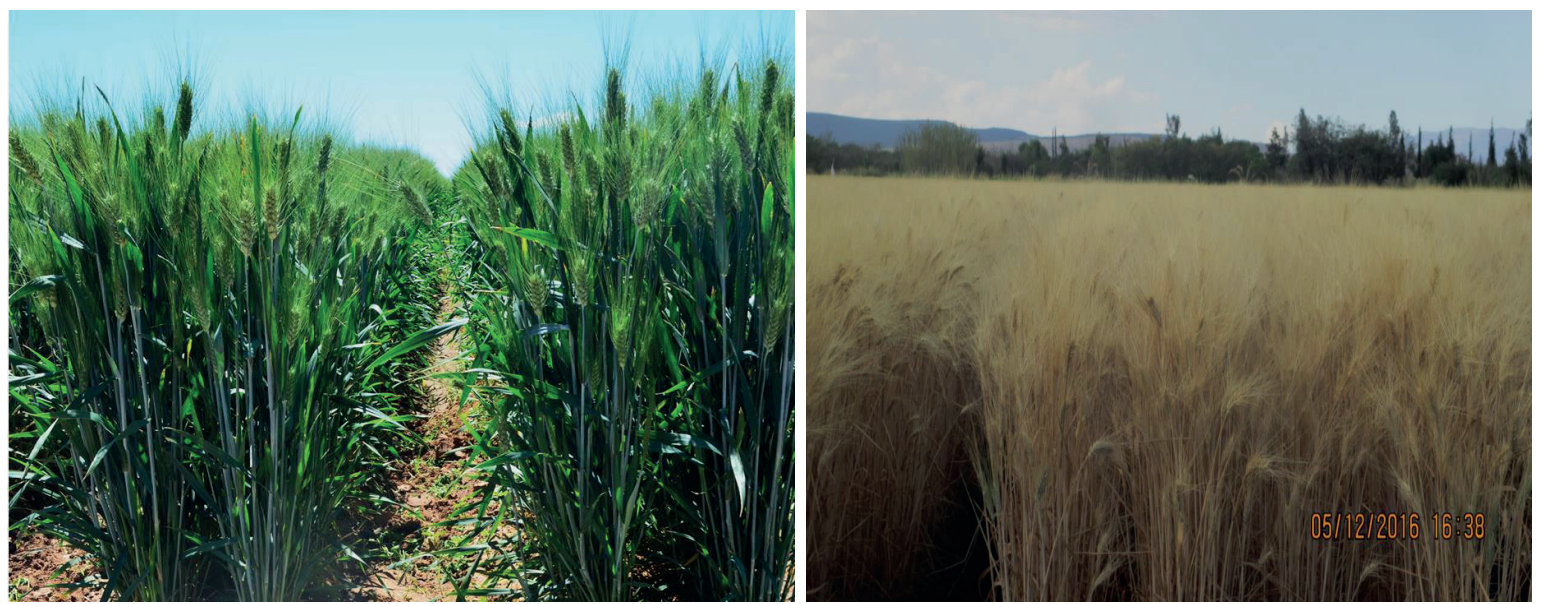

Figura 1. Aspecto de planta de Barobampo C2015.
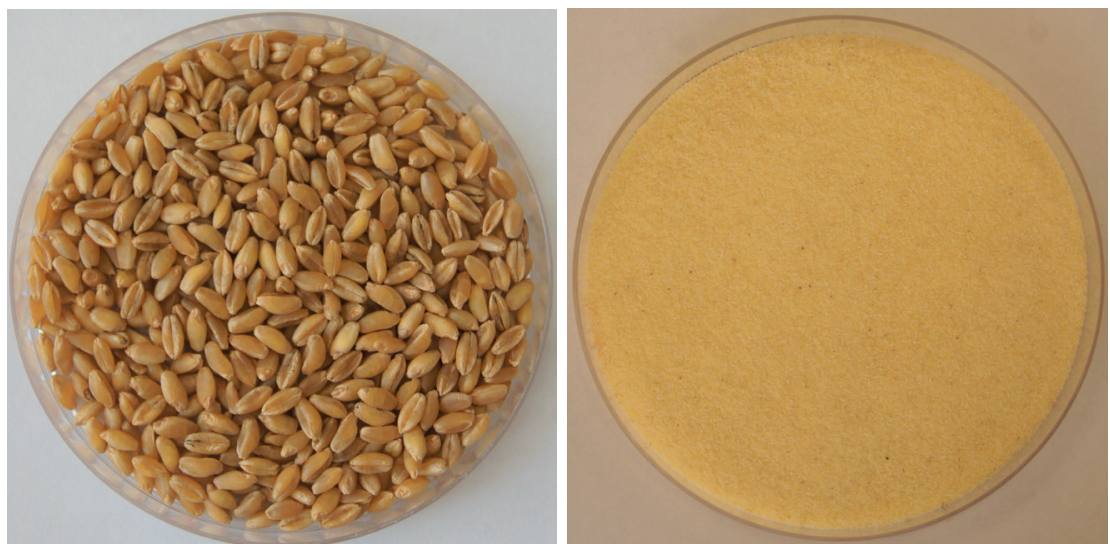

Figura 2. Aspecto del grano vítreo y sémola con pigmentación amarilla de Barobampo C2015. 
de El Bajío se recomienda para siembras tempranas.

La variedad Barobampo C20015 se registró en el Catálogo Nacional de Variedades Vegetales del Servicio Nacional de Inspección y Certificación de Semillas (SNICS) con el registro TRI-158-290916 y cuenta con el título de obtentor Número 1653. Las categorías de semilla original y básica se conservan en los Campos Experimentales Valle de México y Bajío del INIFAP.

\section{AGRADECIMIENTOS}

Al Programa de Trigos Macarroneros del CIMMYT por la generación de la línea y al FONSEC SAGARPA-CONACYT (Proyecto 146788) y MASAGRO Proyecto WYC-2018002 por el financiamiento para el establecimiento de los ensayos nacionales y para la publicación.

\section{BIBLIOGRAFÍA}

CANIMOLT, Cámara Nacional de la Industria Molinera de Trigo (2016) Reporte Estadístico 2015 con Información al 2016. Cámara Nacional de la Industria Molinera de Trigo. Ciudad de México. 116 p.

Pérez-López J. B., E. García-León, H. E. Villaseñor-Mir, R. P. Singh y K. Ammar (2017) Desarrollo de la epifitia de roya de la hoja causada por Puccinia triticina E. en la variedad de trigo cristalino Cirno C2008 durante el ciclo otoño-invierno 2016-2017 en el Bajo Río Mayo, Sonora. Revista Mexicana de Fitopatología 35(Supl. 2017):S39.

SIAP, Servicio de Información Agroalimentaria y Pesquera (2018) Avance de siembras y cosechas. Resumen nacional por estado. Servicio de Información Agroalimentaria y Pesquera, Secretaría de Agricultura, Desarrollo Rural, Pesca y Alimentación. Ciudad de México. http://infosiap.siap.gob.mx:8080/agricola_siap gobmx/AvanceNacionalCultivo.do (Octubre 2018).

Solís M. E., J. Huerta E., P. Pérez H., H. E. Villaseñor M., A. Ramírez R. y M. L. De la Cruz G. (2016) Alondra F2014, nueva variedad de trigo harinero para el Bajío, México. Revista Mexicana de Ciencias Agrícolas 7:1225-1229, https://doi.org/10.29312/remexca.v7i5.248 